
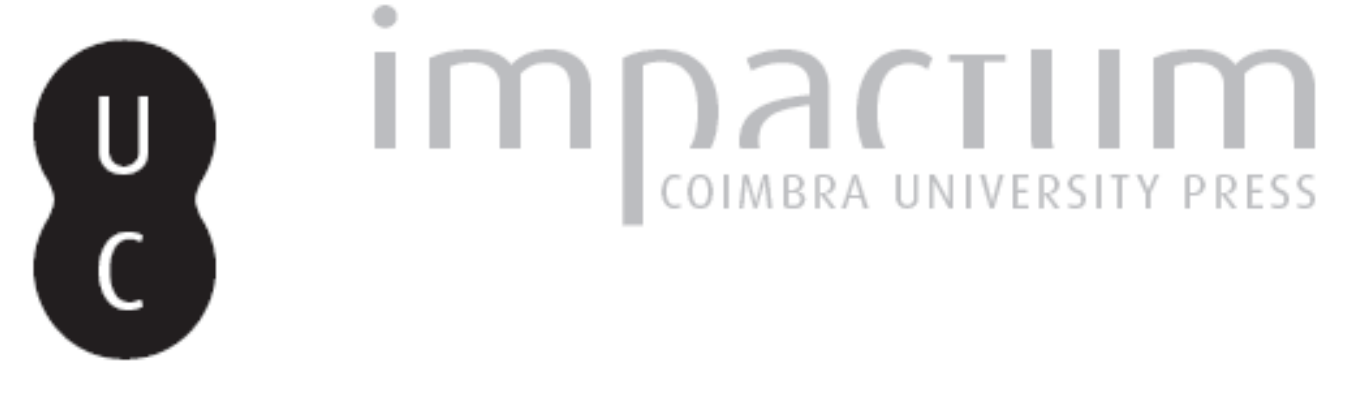

\title{
Religiosità di Verdi
}

\section{Autor(es): $\quad$ Gigliucci, Roberto}

Publicado por: Imprensa da Universidade de Coimbra

URL persistente:

URI:http://hdl.handle.net/10316.2/42430

DOI:

DOI:https://doi.org/10.14195/0870-8584_8_6

Accessed : $\quad$ 26-Apr-2023 12:58:41

A navegação consulta e descarregamento dos títulos inseridos nas Bibliotecas Digitais UC Digitalis, UC Pombalina e UC Impactum, pressupõem a aceitação plena e sem reservas dos Termos e Condições de Uso destas Bibliotecas Digitais, disponíveis em https://digitalis.uc.pt/pt-pt/termos.

Conforme exposto nos referidos Termos e Condições de Uso, o descarregamento de títulos de acesso restrito requer uma licença válida de autorização devendo o utilizador aceder ao(s) documento(s) a partir de um endereço de IP da instituição detentora da supramencionada licença.

Ao utilizador é apenas permitido o descarregamento para uso pessoal, pelo que o emprego do(s) título(s) descarregado(s) para outro fim, designadamente comercial, carece de autorização do respetivo autor ou editor da obra.

Na medida em que todas as obras da UC Digitalis se encontram protegidas pelo Código do Direito de Autor e Direitos Conexos e demais legislação aplicável, toda a cópia, parcial ou total, deste documento, nos casos em que é legalmente admitida, deverá conter ou fazer-se acompanhar por este aviso. 


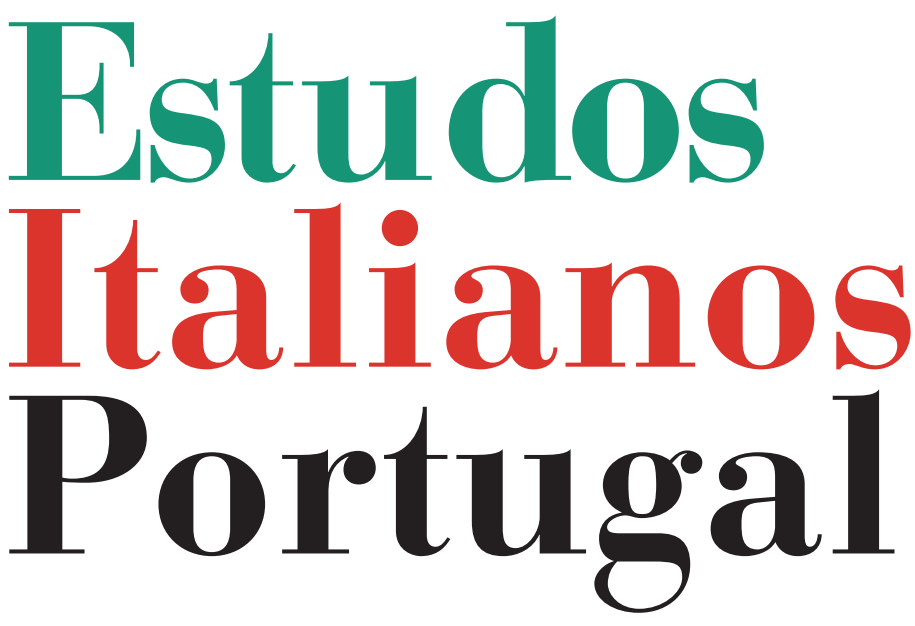

Instituto

Italiano

de Cultura

de Lisboa

Nova Série

$\mathbf{N}^{\circ} \mathbf{8}$ 


\title{
RELIGIOSITÀ DI VERDI
}

\author{
Roberto Gigliucci*
}

QUANDO RADAMÈs, sepolto nella grande tomba, trova Aida, la teatralità del momento è all'apice, un vero trionfo (anti-trionfo della morte sulla marcia trionfale stone-bronzestone-steel-stone), con lo spreco di ingegno scenico potenziale da parte di chi può miscelare l'antico egitto da postkard khedivale alle kafkerie piranesiane. Ma io ho sempre tremato di emozione al pulviscolo aureo che agita il duetto (o quasi) $O$ terra addio, soprattutto con quell'ascesa "e l'alme erranti / volano al raggio dell'eterno dì", sorta di vortice d'aria luminoso di anime alla Dorè.

Mi sono sempre domandato: allora Verdi era religioso? Per il momento non mi interessa la risposta storica, positiva. E poi chi scruta il cuore e le reni dell'uomo al punto, o anche prima del punto? Mi interessa invece il problema della religiosità dell'opera verdiana (e quindi, di nuovo, ma altrimenti, di Verdi).

Proviamo con uno statement: il tema centrale di Verdi, soprattutto del grande Verdi dalla trilogia di metà secolo in avanti, è la stanchezza, il dolore che tende alla fine come

* Roberto Gigliucci insegna alla Sapienza, Università di Roma. Italianista, si occupa anche di comparatistica e del rapporto letteratura-musica. Il suo ultimo volume è Tragicomico e melodramma. Si è occupato anche di lirica petrarchista, di Tasso, di letteratura barocca e di autori novecenteschi quali Montale, Pavese, Croce. E' membro del Centro Interuniversitario Estudos Camonianos. 
congiunzione in un mondo migliore. Questo tropismo indirizzato a un aldilà concerne basicamente le coppie infelici, ma riguarda complessivamente l'universo intero degli infelici, di coloro cui la vita è inferno. Torneremo subito su questo. Consideriamo però prima che Verdi ci offre anche momenti teatrali di religiosità esplicita, di religione, cioè. $\mathrm{Ma}$ sempre anche in questo caso con la notazione intima identitaria religione-pace. Pensiamo obbligatoriamente al duetto del secondo Boccanegra, quello - avrete capito - Vieni a me ti benedico, un inseguirsi atmosferico di due voci l'una grave l'altra tenorile che sono insediate e si sfumano nella pace di quest'ora, un'ora di avemarie severe, una situazione di intervallo, una proiezione verso il tempo antico dove essere pii poteva essere se stessi come umani, una teatralizzazione della voce come casto incanto, sintagma che suona vagamente balordo nella trama verbale del pur grande librettista ma che si annulla nelle sublimi ellissi melodiche dell'invenzione verdiana. Oppure pensiamo - sempre questo invito a pensare, massime perché la musica è un pensiero che si svolge, anche $a$ prescindere dalle parole - a teatremi verdiani fra i più popolari come le scene conventuali, o al miserere de $I l$ trovatore o alle peripezie ispanico-cattoliche della Forza del destino, con fratacchioni e falsi frati, ma ancora e sempre con la Pace esibita subito al massimo grado emotivo vocale della messa di voce (Callas-Caballè nei miei avidi ascolti adolescenziali, scusate se mescolo analisi a memoria, ma come vedremo meglio, per un italiano che parli di Verdi è impossibile altrimenti).

Religione dunque sulla scena, perché efficace melodrammaticamente, ma invece religiosità intima verdiana di una potenza assai più profonda, cui ora torniamo. Che vuol dire religiosità? Molte cose. Non la intendo ora come anelito a Dio e quindi sicurezza (pretesca, intesa nel modo migliore, da parte di chi, scrivente, pretesca ha avuto una educazione) che l'anima di chi nutre questo anelito si salverà. No, non così, non al modo appunto di chi (pretesco, buon prete) si 
incunea nell'agonia di Leopardi o di Verdi e vi intuisce la conversione - vi presume, vi inferisce. No. Religiosità allora come maneggio di cose religiose può essendo totalmente ateo? Del genere: sono materialista assoluto, ma devo scrivere una messa da requiem perché la mia professione di musicista me lo impone. No, neppure questo. Allora che religiosità? Un frisson di percezione metafisica? Ma metafisica non basta. Un frisson teologico? No, questo è superficiale, anche un dozzinante come D'Annunzio poteva averne, di siffatti mormorii spirituali.

Dunque cosa? What are we talking about? Usiamo allora un'altra espressione, quella del sacro. Qui ci ritroviamo in molte occasioni verdiane: andiamo con la mente, per fare un solo esempio, al meraviglioso monologo di Attila Mentre gonfiarsi l'anima e abbiamo la percezione del sacro nell'affabulazione. $\mathrm{O}$ ancora sentiamo rintronare il Dies irae.

Ma pure non ci siamo, o meglio - non ci sono. Il sacro è qualcosa di antropologicamente pervasivo, eterno e incarnato nell'umano primitivo, e non è questo il punto. La religiosità verdiana che vorrei escutere (ed è chiaro a questo punto che il termine religiosità viene qui usato per deficienza di altra terminologia, usato quindi in modo quasi idiolettale) è una questione intima, penetrante e sfuggente. Il modo migliore forse è cercare di coglierla dove la materia narrativa è assolutamente moderna. Nell'unico caso di questo genere, e cioè ne La traviata. Qui forse ci intenderemo meglio.

Violetta ha un attacco di smania malata quando in fine giunge Alfredo al suo letto (divano, tavolo operatorio, ottomana, materazzo spiumato, a seconda della fantasia registica) e lei è stravolta da pulsioni e pensieri violentissimi. Prima un mancamento, fu debolezza, poi ecco la smania: uscire, uscire, fuori dove sacrificano idealmente il bue grasso infiorato, il quadrupede vestito a festa per il macello, come nelle immaginose pagine di Frazer, ma trasportate nella Parigi crowded desert. Inutile dire che quella carnascialesca vacca 
da squartare è Violetta stessa, sacrificale quante altri mai. Ma torniamo dentro l'appartamento di Violetta (che Zeffirelli spossessava del suo mobilio in una melanconia di declino economico). Lei vuole abbigliarsi, ma ricade spossata, esattamente non può, la tisi non le accorda che poche ore, pochi minuti in scena. Allora succede qualcosa nella dimensione teatro-musicale verdiana, qualcosa di religioso. Alfredo, contorto dentro, manda per il dottore e Violetta smaniosa e preda di venti psichici in conflitto dice che sì, Annina vada dal dottore a dirgli che è ritornato l'amore, è ritornata la vita, e dicendo questo lei avverte che la vita si dilegua e fugge, ma ecco, infinitamente grande invenzione verdiana, ecco che arriva quell'avversativa, "Ma. Ma se tornando non m'hai salvato,/a niuno in terra salvarmi è dato". Qui rintocca la religiosità. Qui almeno io sento un avvertimento della presenza della morte (qualche regista potrebbe metterla addirittura in scena, la morte, ma non so come, sarebbe sicuramente un pasticcio kitsch, o forse no) che travalica ogni amore, o meglio proietta ogni amore melodrammatico in uno scenario di aldilà, ma non precisamente di aldilà istituzionale: un aldilà che probabilmente non c'è in senso religioso canonico ma esiste nella prospezione teatrale, ma nemmeno un aldilà finto, una metafisica di cartapesta, perché è un aldilà che avverte Verdi per primo, un sentimento del trapasso in senso pieno che sgomenta ma chiude, forse chiude, gran dio. Dopo c'è l'ascensione delle note sul pentagramma e la pseudo-cabaletta a due, un'antitesi rispetto a Parigi o caralo. Ma prima, oh, prima c'è ben altro. C'è questa cosa che chiamo insufficientemente religiosità verdiana. $\mathrm{E}$ il fatto davvero ancor più di rilievo è che qui la religiosità (il rintocco, l'avvertimento, l'altissimo) è insediata nel décor contemporaneo, squallido, nella stanza di una cocotte pur mezza santa (ben altro naturalismo crudele era quello del romanzo di Dumas figlio, escludendo la posteriore riduzione in pièce) ma anche ormai mezza matta, sia detto con religioso dolore e serietà. Prima 
della progressione ascendente e del Gran dio morir sì giovane, che in fondo nella sua drammaticità è uno sfogo dall'insostenibile, c’è l'agglomerarsi della religiosità come angoscia nel registro grave.

Tutta La traviata, che eleva il linguaggio al massimo grado proprio ove la materia è più bassa del solito (memorabilmente lo ha illustrato Baldacci), ospita queste occasioni in cui Violetta si ritrova dentro gorghi di solitudine anche in mezzo alla festa, anzi soprattutto in mezzo alla festa. Ad esempio in quella di Flora, durante la scena delle carte: l'a solo di Violetta, "che fia morir mi sento" (che maledettamente e contro ogni indicazione di partitura, come si desume anche dall'edizione critica, svariati direttori stolidi rallentano con insipienza drammaturgica). Si tratta di uno spasmo dentro al popoloso deserto, un senso del fato, si potrebbe dire in termini pagani, ma io direi piuttosto un'altra marca religiosa, non certo della santità o pietà dell'eroina, ma nel senso di una sacralità intima quale avvertimento del rintocco, più che paura dello scandalo incombente, una richiesta di pace impossibile nonostante che Violetta abbia operato sacrificalmente per la pace, ma è stato vano, tutto è frainteso quaggiù, in questo avello che chiamano vita, e vita sociale nella fattispecie.

Si ritorna così all'avello visualizzato dell' $A$ ida, e all'invocazione alla pace con doppia forcella $(<>)$ di Leonora della Forza, quindi alla conclusione della Forza stessa, ma anche all'aria di Alvaro, dell'esule che peraltro invano si cela al mondo (invano Alvaro, quasi un bisticcio) e quindi si evochi anche quel magnifico Le minacce e i fieri accenti che segna l'opera fin dalla ouverture ed è il sigillo più evidente dell'invano, ma non perché il destino sia il potere cogente, ma perché invano è l'aldiquà, tutto è invano sulla terra, e quindi tutti si proiettano verso una dimensione altra, ove non sia invano. Ma anche qualora questa dimensione sulla scena sia formalizzata come istituzionalmente religiosa, la religiosità di cui qui parliamo resta assolutamente non istituzionale. 
Ma lassù ci vedremo in un mondo migliore, il duetto di Carlo e Isabella sul prefinale del Don Carlo, sembrerebbe una trita e quasi triviale declinazione del confidare cattolico nella salvezza. Nulla di tutto questo. L'aspirazione alla pace, l'invanità (ci si perdoni il termine) dell'amore fra i protagonisti eterosessuali, l'invanità dell'amore tra i protagonisti omosessuali (Carlo e Rodrigo), l'invanità del potere, tutto è invano quaggiù, persino le note gravissime che accompagnano l'inquisitore e lo sfarzo dell'autodafé sono parte di una invanità che è il teatro verdiano in complesso; l'aspirazione alla pace è la verbalizzazione di questa religiosità paradossale, che scorre parallela al tema religioso istituzionale e quasi ne è un contraltare (senza altare) segnando una verità che nessun paramento potrà mai rappresentare.

La religiosità verdiana, in tale senso complesso che fatico molto a definire, emerge persino contro le parole dei librettisti, certo contro quelle di Boito: nel finale fugato del Falstaff ciò che in Boito è irrisione di gusto elisabettiano, Tutto nel mondo è burla, diventa con l'intonazione di Verdi un qualcosa di tremendamente religioso, con quella pausa dopo la risata final, così pericolosamente vicina a luoghi del Requiem. Siamo in una dimensione mozartiana, pensando cioè a cosa fa Mozart delle parole di Da Ponte nella scena della statua a cena: un terzetto comico (al limite serio-comico, ma in un dramma giocoso) peraltro rubacchiato molto bene da Bertati, com'è noto, diventa la scena primaria di ogni teatro di tutti i tempi, la scena dell'uomo difronte a un maestoso ostacolo metafisico. Quell'ostacolo punitivo è ingiusto? O è giusto? La domanda resta aperta.

Certo il paragone con Mozart del 1787 è troppo arrischiato. Ma intendo dire che la religiosità verdiana (l'attributo paradossale sia dato ormai per implicito) è troppo forte per perdersi nell'estremo tentativo ultrariuscito di comporre finalmente una seconda opera comica. Nel Falstaff tutti riconoscono parodie di precedenti melodrammi dello stesso 
autore; si rammenti solo quel povera donna che ci riporta alla nostra Violetta. Dunque comicità, e ancora dentro il comico le tracce della religiosità verdiana, che quindi credo sia ormai chiaro non aver niente a che fare con la religiosità intesa nel senso più comune. Del resto l'ultima opera non è un exploit di commedia che non sia preceduto da sperimentazioni in quel senso. Si impongono le ovvie scene buffe o grottesche della Forza, che fanno di quest'opera uno dei calderoni più mescidati nella carriera di Verdi. Poi la mente va subito al Ballo in maschera e alla crudele derisione della protagonista svelata, con la risata degli astanti, o ancora all'ambivalenza del personaggio di Oscar eccetera. Ma si risale benissimo pure al Rigoletto, dove la comicità è nell'abbassamento dei registri linguistici dentro più dentro dove l'orrore è orrore: "Rattoppa quel sacco... Uccider quel gobbo!/che diavol dicesti" e così via. Qui nell'incalzare stupefacente del senario che diventa il ritmo preciso e ostinato dell'inferno (se ne ricorderà Puccini coi suoi scrittori nell'atto secondo della Tosca, quasi un film di Costa-Gavras: "Lo strazia quel vostro/silenzio assai più" e dintorni), la bassezza e la sublimità sacrificale sono fusi in un unico battito, in una quasi infinita percussione di palpito e di omicidio.

Ma forse la religiosità di cui parlo è discernibile in modo davvero eclatante (pensando a éclat, quindi lampante e talora radiante) ne $I l$ trovatore, che con una storia così vecchia e un libretto così tradizionale (rispetto ai coevi capolavori verdiani, e considerando che Il trovatore è la parodia seria della Lucia di Cammarano-Donizetti) si verticalizza di continuo verso celestialità. Celestialità in mezzo all'animalità $o$ brutalità: sembra un pattern molto collaudato e banale nel melodramma dell'Ottocento, ma con Il trovatore c'è molto di più sul piano semantico. Sei tu dal ciel disceso è l'isolamento mistico di Leonora, isolamento che contempla l'unione con Manrico, ma la proietta - ancora - verso un altrove alto, altissimo, inarrivabile forse. $O$ in ciel son io con te, questa frase è 
un ombelico dell'opera, una frazione di senso che racchiude il senso intero. In un'opera oscura e notturna, dove la luna è una madre falsa-madre omicida più che la mamma di Meleagro, e anzi una madre assassina che orchestra in modo registico la vendetta, quasi uno Jago in abito da zingara, in questo tenebrore insomma che la luce selenica oscura vieppiù, la luce che illumina è intravista in alto e oggetto di anelito fondamentale. Manrico, che ha un braccio sempre dichiarato fortissimo e in realtà debolissimo, può solo in ciel precedere Leonora, è quasi un suo fragile seguace in questa torsione verso l'alto, verso una luce irraggiante e forse impossibile, ma chiave di tutto, questo è certo.

$\mathrm{Ci}$ si può rivedere nell'estasi, raggianti di splendore, cantano gli eroi verdiani, a voler dire che c'è una tensione verso la fulgenza assoluta, ma di narrato e inscenato c'è solo la religiosità intima individuale o di coppia che induce a guardare a un aldilà.

Senza crederci? Questo non è poi così importante, un'ultima nota tenuta fino all'acuto pianissimo può bastare per chiarire che la musica, sempre semantica e mai "assoluta" come il formalismo ci vuole far credere per pigrizia interpretativa, lascia nell'ascoltatore solo inquietudine, o meglio tensione verso il significato. L'offertorio del Requiem è un bel pezzo di melodramma verdiano che ci sprofonda in questa religiosità paradossica, più piena e flagrante certo del Rossini sacro, che pure nel Quando corpus a cappella esprimeva una interrogazione sorprendente e suprema (diavolo di un Rossini, modello per tutto il serio e tutto il comico dell'opera del XIX secolo). Il cecidere manus di Manzoni certo qui sarebbe pertinente, ma non voglio giammai ridurre la religiosità verdiana a l'espressione mera di un dubbio religioso (nel senso comune del termine). I dubbi metafisici di Verdi qui non sono affatto in questione, pur essendo interessante oggetto di ermeneusi. Io credo di aver parlato, o solo sussurrato, a proposito di una religiosità altra, paradossale, ardua da cogliere. 
A un italiano, e per di più con la tara di italianista, come chi scrive, Verdi appare soprattutto come il grande unico nostro artista ottocentesco che semina le marche di una religiosità quasi indefinibile nei suoi capolavori. Meglio di Manzoni, Verdi in questo senso esprime un pessimismo che è lontanissimo anche da quello di Verga o di Flaubert, così come è sideralmente distante dalla super tensione annichilante del Tristan, o meglio dalla sua propensione sfrenata alla disidentificazione, alla prolungata perenne e sempre finale ekstasis.

La religiosità semiterrena antiterrena di Verdi sembra invece quasi tanto imprendibile quanto incoercibile, così forte da travestire in lutto le zingarelle e il canto carnascialesco di La traviata. Semplicemente perché a nessuno in terra salvarci è dato. 